\title{
Folate intakes from diet and supplements may place certain Canadians at risk for folic acid toxicity
}

\author{
Adriana N. Mudryj ${ }^{1 *}$, Margaret de Groh ${ }^{2}$, Harold M. Aukema ${ }^{1}$ and Nancy $\mathrm{Yu}^{1,3}$ \\ ${ }^{1}$ Human Nutritional Sciences, University of Manitoba, Winnipeg, MB, Canada R3T 2N2 \\ ${ }^{2}$ Public Health Agency of Canada, Ottawa, ON, Canada K1A OKO \\ ${ }^{3}$ Community Health Sciences, University of Manitoba, Winnipeg, MB, Canada R3T 2N2 \\ (Submitted 14 April 2016 - Final revision received 27 July 2016 - Accepted 1 August 2016 - First published online 9 September 2016)
}

\section{Abstract}

To examine the prevalence of folate inadequacy and toxicity based on usual intakes from food and supplements, as well as biomarkers of folate, secondary data analyses were performed using cross-sectional, nationally representative data from the Canadian Community Health Survey, Cycle 2.2 ( $n$ 32 776), as well as biomarker data from the Canadian Health Measures Survey, Cycles 1,2 and 3 ( $n$ 15 754). On the basis of unfortified food sources, Canadians would struggle to consume adequate amounts of folate. When folate intakes from all food sources were considered, the overall prevalence of folate inadequacy was low across all age/sex groups, with the exception of females $>70$ years. However, $>10 \%$ of supplement users were above the tolerable upper intake level, increasing to almost $18 \%$ when overage factors were accounted for. In addition, between 20 and $52 \%$ of supplement users had elevated erythrocyte folate concentrations, depending on the cut-off used. Results from this study suggest that insufficient dietary intakes of folate in Canadians have been ameliorated because of the fortification policy, although folate inadequacy still exists across all age groups. However, supplement users appear to be at an increased risk of folic acid (FA) overconsumption as well as elevated erythrocyte folate. As such, the general population should be informed of the potential risks of FA overconsumption resulting from supplement use. This study suggests a need for more careful assessment of the risks and benefits of food fortification, particularly fortification above mandated levels, and FA supplement use in the general population.

\section{Key words: Folate: Food fortification: Health surveys: Supplements}

Folate (vitamin $\mathrm{B}_{9}$ ) is a water-soluble vitamin, necessary for cell division and growth, which occurs naturally in foods such as leafy green vegetables, fruits and legumes. Its synthetic form, folic acid (FA), is found in supplements and fortified foods ${ }^{(1)}$. Folate deficiency during the periconceptional period (3-4 weeks after conception) has been associated with an increased risk for neural tube defects (NTD) such as spina bifida and anencephaly ${ }^{(2-4)}$, and several studies have corroborated the effect of FA supplementation on lowering the prevalence of NTD and other birth defects $^{(5-7)}$. As such, adequate intakes of folate are essential for women of childbearing age (WCBA), and the Institute of Medicine (IOM) recommends that WCBA consume $400 \mu \mathrm{g}(0 \cdot 4 \mathrm{mg})$ a day of FA in addition to consuming foods naturally rich in folate ${ }^{(8,9)}$. In 1996, the United States Food and Drug Administration announced that it would allow for the fortification of flour and other cereal grain products with FA, deeming fortification mandatory in 1998. The very same year, Canada also mandated the addition of FA to white flour and enriched pasta and maize meal at $0.15 \mathrm{mg}$
$\mathrm{FA} / 100 \mathrm{~g}$ of flour and $0.20 \mathrm{mg} \mathrm{FA} / 100 \mathrm{~g}$ of pasta $^{(9)}$, predicting an overall intake increase of $100 \mu \mathrm{g} / \mathrm{d}^{(10)}$. After fortification, results showed a dramatic decrease (approximately 40\%) in national rates of NTD $^{(11,12)}$ and an improvement in folate status ${ }^{(13,14)}$.

In spite of this success, there exists some controversy regarding possible health concerns associated with FA overconsumption. The tolerable upper intake level (UL) is defined as the 'maximum level of chronic nutrient intake that is likely to pose no risk of adverse health effects to almost all individuals in the general population, ${ }^{,(15,16)}$. On the basis of the metabolic interactions between folate and vitamin $\mathrm{B}_{12}$, the Food and Nutrition Board (FNB) and the IOM established an UL for synthetic forms of folate ( $\mu \mathrm{g}$ of FA) available in dietary supplements and fortified foods for each life-stage group ${ }^{(1)}$.

Recently, FA fortification has come under scrutiny because of accumulating evidence suggesting that the general population of the USA has been exposed to unprecedented levels of FA above the $\mathrm{UL}^{(17)}$. This may be attributed to the fact that high

Abbreviations: CCHS 2.2, Canadian Community Health Survey, cycle 2.2; CHMS, Canadian Health Measures Survey; DFE, dietary folate equivalent; DRI, dietary reference intakes; EAR, estimated average requirement; FA, folic acid; IOM, Institute of Medicine; NHANES, National Health and Nutrition Examination Survey; NTD, neural tube defects; POFI, prevalence of folate inadequacy; UL, tolerable upper intake level; WCBA, women of childbearing age. 
plasma folate has been associated with an exacerbation of both clinical and biochemical signs of $\mathrm{B}_{12}$ deficiency, potentially permitting cognitive impairment to occur ${ }^{(18,19)}$. A recent review article outlined the increased risk of anaemia and cognitive issues seen in older adults with high serum folate but poor $\mathrm{B}_{12}$ status, implying that excessive FA intake is not safe and is associated with adverse clinical outcomes in the elderly ${ }^{(18,19)}$. This is of particular concern in older adults who are more susceptible to vitamin $\mathrm{B}_{12}$ deficiency ${ }^{(20)}$. However, older adults are not the only ones at risk of adverse health outcomes: an increase in insulin resistance has been linked to high maternal erythrocyte folate among Indian mothers ${ }^{(21)}$. In addition, animal studies have shown that excessive FA may be harmful, reducing natural killer cell cytotoxicity in mice ${ }^{(22)}$ and also disturbing their immune response and resistance to malaria ${ }^{(23)}$. Trends also suggest that after fortification, North America has experienced a 'reversal' of the downward trend in the incidence of colorectal cancer at a statistically significant rate of 4-6 cases/ 100000 individuals, although causality cannot be inferred ${ }^{(24)}$. However, even though there is evidence that excess FA may be associated with advanced colorectal adenomas ${ }^{(25)}$ and recurrent colorectal adenomas $^{(24,26)}$, a recent meta-analysis study does not support this ${ }^{(27)}$. Nevertheless, results from the Prostate, Lung, Colorectal, and Ovarian Cancer Screening trial observed that women who reported consuming $\geq 400 \mu \mathrm{g} / \mathrm{d}$ of FA had a $20 \%$ greater risk of developing breast cancer ${ }^{(28)}$. The hypothesised mechanism behind these phenomena suggests that, although FA aids in normal DNA replication, in malignant cells excess amounts may cause cell proliferation ${ }^{(29)}$. As it passes through the intestinal wall, FA is converted to a natural biological form of the vitamin, resulting in the circulating form 5-methyltetrahydrofolate ${ }^{(30,31)}$. It has been shown that doses of FA in physiological quantities can 'saturate' this conversion mechanism, resulting in measurable levels of circulating FA, which may be detrimental ${ }^{(31)}$.

Mandatory fortification has resulted in increased exposure to circulating $\mathrm{FA}^{(17)}$, and it has been observed that increased concentrations of plasma FA in elderly women who took FA-containing supplements $(>400 \mu \mathrm{g}$ ) were inversely associated with decreases in the cytotoxicity of circulating natural killer cells ${ }^{(32)}$. Although the biochemical and physiological consequences of overconsumption remain unclear, there remains concern over the adverse effects of high levels of $\mathrm{FA}^{(24,33)}$. Previous studies have indicated that it is permissible for manufacturers to fortify at higher levels than the mandated amount, and Shakur et al. have estimated that the amount of FA in Canada's fortified foods may be as high as $50 \%$ more than the quantity expected based on the government-authorised levels ${ }^{(34-36)}$. Thus, it is critical not only to observe the dietary intake of folate but also to measure erythrocyte folate concentrations to obtain a clearer picture of the folate status of the Canadian population in a post-fortification era. The aim of this study was to determine whether there are groups at risk for toxicity or deficiency of this vitamin. In addition, we assessed the intake of supplement users separately from non-supplement users, as this particular group could be at an increased risk of FA overconsumption as well as elevated levels of erythrocyte folate $^{(37,38)}$

\section{Methods}

\section{Data source}

This study used data from the Canadian Community Health Survey, Cycle 2.2 (CCHS 2.2) 2004, as well as data from the Canadian Health Measures Survey, Cycles 1, 2 and 3 (CHMS). In total, the CCHS 2.2 surveyed 35107 respondents, each of whom completed a general health questionnaire as well as a 24-h dietary recall, administered by trained interviewers. A subsample of 10786 respondents completed a second 24-h dietary recall, 3-10 d later ${ }^{(39)}$. This study utilised the CCHS 2.2 Share File, restricting the analysis to the $95.3 \%$ respondents who agreed to share their information for research purposes ${ }^{(39,40)}$. Further details on the CCHS 2.2 survey methodology and sampling design can be found elsewhere ${ }^{(40)}$. In addition to demographic details, the general health component of the CCHS 2.2 captured information on vitamin and mineral supplement use during the $30 \mathrm{~d}$ before the interview $^{(41)}$. Exclusion criteria for this study included women who were pregnant or breast-feeding at the time of the survey, those who had an invalid or missing dietary recall, those who reported consuming only breast milk and those who did not report any food items consumed during the 24 -h recall period, resulting in a final sample size of $n 32776$. CCHS 2.2 data were used for folate intake analysis.

The CHMS is a comprehensive direct health measures survey, which includes blood, urine and anthropometric measures and banks specimens for future measurements and genetic research. The CHMS has collected data in three cycles (2007-2009, 2009-2011 and 2012-2013) with a minimum of 500 respondents for each sex from 10 age groups of Canadians - 6-79 years (Cycle 1) and 3-79 years (Cycles 2 and 3). Cycles 1, 2 and 3 surveyed 5604, 6395 and 5785 respondents, respectively. The CHMS also captured the intake of vitamin and mineral supplements $30 \mathrm{~d}$ before the clinic visit ${ }^{(42,43)}$. CHMS data were used for the frequency of supplement use and erythrocyte folate level estimates.

\section{Folate intake estimates}

In order to determine folate consumption habits of Canadians, folate intake was categorised as follows: naturally occurring folate from foods (food folate) and the synthetic form from supplements or from fortification (FA). The dietary folate equivalents (DFE) for synthetic FA sources were calculated, a method introduced in 1998 by the FNB and IOM to take into account the higher bioavailability of synthetic FA compared with natural folate ${ }^{(44)}$. The DFE for specific foods was calculated as the amount of food folate $(\mu \mathrm{g})$ plus 1.7 times the amount of synthetic FA $(\mu \mathrm{g})$ from the $24-\mathrm{h}$ dietary recall, thus taking into account both natural folate and synthetic FA. As supplements taken in the CCHS 2.2 were assumed to have been consumed on an empty stomach, the aforementioned calculation was modified for supplemental FA, resulting in a conversion factor of 2. The DFE values for natural folate and FA from food fortification were also kept as separate values to estimate intake from these different sources.

Folate intakes were calculated for separate dietary reference intakes (DRI) life-stage groups by FA supplement use (total folate intake from all sources was also calculated). In addition, 
the amount of folate from fortified sources was re-calculated with an 'overage' factor, defined as the 'potential extra amount of FA added to a product during fortification by the food manufacturer to prevent decay/loss during shelf life/storage ${ }^{,(36)}$. For key food groups (breads, baked goods, etc.), the amount of folate in the product, plus overage, was calculated by multiplying initial amounts by an adjustment factor based on the food group category $^{(36)}$. Folate intakes for the three sources and for all the sources, combined, were then compared with the estimated average requirement (EAR) for DRI life-stage groups, which is the amount of a nutrient expected to meet the needs of $50 \%$ of the population, and the $\mathrm{UL}^{(15,16)}$. Nutrient and food intake data were analysed using SAS software, version 9.1 (SAS Institute Inc.). Version 1.11 of the Software for Intake Distribution Estimation (SIDE)-IML program was used in conjunction with SAS to generate an estimation of the usual dietary intake by using both the first and the second dietary recall ${ }^{(45,46)}$ along with bootstrapping weights to estimate variance.

\section{Blood folate estimates}

A total of three cycles of CHMS data were pooled to provide a larger data set for statistical evaluation of erythrocyte folate analysis. Biomarker analysis is described briefly in this study and in detail elsewhere ${ }^{(47)}$. Blood samples for erythrocyte folate analysis were collected by a certified phlebotomist. Erythrocyte folate content was measured using the Immulite 2000 assay

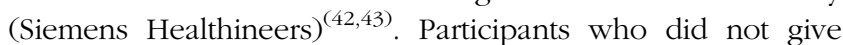
blood or had unusable samples were excluded from the analysis, as were respondents under 6 years of age, to ensure compatibility between cycles (only Cycles 2 and 3 had participants aged 3 years and above), resulting in a final sample size of 15754. SPSS, version 22, and STATA 13 software were used to generate an estimation of Canadians with elevated erythrocyte folate concentrations along with bootstrapping weights to estimate variance, by life-stage group as well as by supplement use. Erythrocyte folate status was assessed using cut-off values for elevated blood levels that have been proposed in the literature.

Recently, it has been suggested that erythrocyte folate concentrations differ depending on the assay used, depending on certain variations or calibrations ${ }^{(48)}$. Using a sample of 152 individuals and two different assays (Immulite 2000 and the microbiological assay), Colapinto et al. used the Deming regression method to create the following equation:

Predicted microbiological assay concentration $=-22 \cdot 95$

$$
\times(0 \cdot 81) \times \text { Immulite } 2000 \text { assay concentrations. }
$$

Given that results from differing assay methods can be large, and that the microbiological assay is deemed the gold standard for determining erythrocyte folate concentrations, it seemed prudent to utilise the adjustment method proposed by Colapinto to convert Immulite 2000 assay results to those on par with the microbiological assay used in the National Health and Nutrition Examination Survey (NHANES) or 'NHANES level'. However, the results must be interpreted with caution, as converting CHMS Immulite 2000 assay to microbiological assay values lowers erythrocyte folate concentrations, and thus may under-represent the number of Canadians who have elevated levels of erythrocyte folate.

\section{Results \\ Overall dietary folate equivalent intake}

The average DFE intake for Canadians, excluding supplements, was $442 \mu \mathrm{g}$. When overage factors were added to the total DFE, intake increased to $487 \mu \mathrm{g}$. The highest intakes were seen among males aged 14-50 years, ranging from 521 to $576 \mu$ g (Table 1). Among all life-stage groups, average DFE intakes surpassed the EAR values, irrespective of the calculation method. When DFE intake was calculated with the addition of FA from dietary supplements, the overall intake was significantly higher for the supplement user group $(P<0.001)$ compared with nonsupplement users by a difference of approximately $900 \mu \mathrm{g}$. Intake was also significantly higher among supplement users in all age/ sex groups when compared with non-supplement users in the same group (Table 1). Overall, non-supplement using WCBA had mean DFE intakes of 417 (SE 113) $\mu \mathrm{g}$, whereas women who consumed supplements had an intake of 1474 (SE 501) $\mu \mathrm{g}$.

\section{Supplemental folate use}

Both CCHS 2.2 and CHMS data showed comparable proportions of Canadians who take FA-containing supplements (Table 2). Overall, the CCHS reported that $25 \%$ of Canadians consumed a supplement containing FA, ranging by age group from 13 to $38 \%$. Slightly lower results were observed by age group using the combined CHMS cycles (22\%). The highest user rate defined by the CCHS was found in children aged $4-8$ years (38\%), and the lowest rate of supplement use occurred among males aged 14-18 years (13\%). The CHMS also found high rates among children $<8$ years (29\%) as well as in females $>70$ years (38\%). In all, $23 \%$ of males and $28 \%$ of females reported use of a supplement containing FA in the CCHS and $19 \%$ and $25 \%$ reported supplement use in the CHMS, respectively. With the exception of males aged 9-13 years, females in every other age group had higher rates of FA supplement use according to CCHS 2.2 data. Similar patterns were found using CHMS pooled cycles, with females reporting higher supplement use in every age group with the exception of those under 8 years. Females $>31$ years reported higher supplement use proportion than the national average. CHMS data observed the same trend (data not shown). Overall, comparable proportions were reported by WCBA (24\% in the CCHS and almost $23 \%$ in the CHMS) and in the general population (26\% in the CCHS and $22 \%$ in the CHMS).

\section{Assessment of the prevalence of folate inadequacy: dietary folate}

Folate inadequacy among Canadians was calculated by measuring the proportion of individuals in each DRI life-stage group who did not meet EAR criteria for folate (Fig. 1(a)). Overall, the prevalence of folate inadequacy (POFI) was low $(<6 \%)$ among female respondents $<13$ years and males $<50$ years, regardless of the calculation method or supplement use status. 
Writish Journal of Nutrition

Table 1. Folate intake of Canadians by source and supplement use based on results from the Canadian Community Health Survey, cycle 2.2 (Mean values with their standard errors)

\begin{tabular}{|c|c|c|c|c|c|c|c|c|c|c|}
\hline \multirow[b]{2}{*}{ Life-stage groups } & \multicolumn{2}{|c|}{ Natural folate } & \multicolumn{2}{|c|}{ DFE } & \multicolumn{2}{|c|}{ DFE + overage $\ddagger$} & \multicolumn{2}{|c|}{ Folic acid } & \multicolumn{2}{|c|}{ Folic acid + overage } \\
\hline & Mean & SEM & Mean & SEM & Mean & SEM & Mean & SEM & Mean & SEM \\
\hline \multicolumn{11}{|l|}{ Overall ( $n 32776$ ) } \\
\hline $1-3$ years $(n 2117)$ & 144 & 59 & 280 & 103 & 320 & 117 & 114 & 76 & 137 & 83 \\
\hline $4-8$ years $(n$ 3235) & 173 & 59 & 381 & 105 & 444 & 114 & 162 & 72 & 196 & 79 \\
\hline Male: $9-13$ years $(n$ 2080) & 213 & 69 & 466 & 123 & 541 & 136 & 182 & 81 & 222 & 90 \\
\hline Female: $9-13$ years $(n 1980)$ & 181 & 58 & 403 & 108 & 470 & 119 & 161 & 81 & 196 & 88 \\
\hline Male: $14-18$ years ( $n$ 2288) & 255 & 98 & 563 & 182 & 651 & 208 & 231 & 131 & 275 & 144 \\
\hline Female: $14-18$ years ( $n$ 2256) & 198 & 77 & 432 & 150 & 495 & 177 & 198 & 136 & 231 & 148 \\
\hline Male: $19-30$ years $(n$ 1804) & 272 & 101 & 576 & 171 & 653 & 189 & 259 & 170 & 298 & 181 \\
\hline Female: $19-30$ years $(n 1854)$ & 210 & 82 & 410 & 124 & 456 & 133 & 232 & 216 & 254 & 219 \\
\hline Male: $31-50$ years $(n$ 2596) & 262 & 90 & 521 & 155 & 588 & 173 & 241 & 197 & 279 & 209 \\
\hline Female: $31-50$ years $(n 2686)$ & 228 & 99 & 406 & 137 & 446 & 147 & 279 & 286 & 300 & 285 \\
\hline Male: $51-70$ years $(n 2550)$ & 263 & 109 & 463 & 155 & 515 & 169 & 279 & 276 & 305 & 281 \\
\hline Female: $51-70$ years $(n 3200)$ & 223 & 82 & 381 & 113 & 418 & 125 & 281 & 329 & 300 & 329 \\
\hline Male: 71 years and over $(n 1520)$ & 224 & 87 & 394 & 123 & 447 & 139 & 267 & 305 & 292 & 309 \\
\hline Female: 71 years and over $(n 2610)$ & 195 & 75 & 327 & 102 & 363 & 110 & 268 & 351 & 286 & 352 \\
\hline Overall $(n 32776)$ & 230 & 96 & 442 & 160 & 487 & 181 & 217 & 215 & 274 & 238 \\
\hline \multicolumn{11}{|l|}{ Supplement users $(n$ 8390)§ } \\
\hline $1-3$ years $(n 603,28 \%)$ & 163 & 61 & $523^{\star \star \star}$ & 168 & $566^{\star \star \star}$ & 169 & $191^{\star \star \star}$ & 73 & $215^{\star \star \star}$ & 74 \\
\hline $4-8$ years $(n 1243,38 \%)$ & 171 & 54 & $580^{\star \star *}$ & 162 & $643^{\star \star \star}$ & 170 & $221^{\star \star \star}$ & 73 & $254^{\star \star *}$ & 79 \\
\hline Male: $9-13$ years $(n 462,22 \%)$ & 218 & 64 & $728^{\star \star \star}$ & 256 & $810^{\star \star \star}$ & 264 & $275^{\star \star \star}$ & 120 & $317^{\star \star \star}$ & 128 \\
\hline Female: $9-13$ years $(n 425,22 \%)$ & 196 & 54 & $694^{\star \star \star}$ & 302 & $767^{\star \star \star}$ & 308 & $271^{\star \star \star}$ & 146 & $308^{\star \star \star}$ & 155 \\
\hline Male: $14-18$ years $(n 293,15 \%)$ & 266 & 91 & $1227^{\star \star \star}$ & 586 & $1318^{\star \star \star}$ & 603 & $502^{\star \star \star}$ & 272 & $548^{\star \star \star}$ & 282 \\
\hline Female: $14-18$ years ( $n 340,13 \%)$ & 223 & 69 & $1178^{\star \star \star}$ & 638 & $1249^{\star \star \star}$ & 640 & $501^{\star \star \star}$ & 301 & $537^{\star \star \star}$ & 301 \\
\hline Male: $19-30$ years $(n 313,15 \%)$ & 290 & 112 & $1497^{\star \star \star}$ & 484 & $1585^{\star \star \star}$ & 519 & $632^{\star \star \star}$ & 231 & $678^{\star \star \star}$ & 250 \\
\hline Female: $19-30$ years ( $n 452,24 \%)$ & 213 & 70 & $1328^{\star \star \star}$ & 652 & $1370^{\star \star \star}$ & 645 & $578^{\star \star \star}$ & 326 & $599^{\star \star \star}$ & 327 \\
\hline Male: $31-50$ years $(n 541,21 \%)$ & 263 & 81 & $1451^{\star \star \star}$ & 652 & $1517^{\star \star \star}$ & 667 & $616^{\star \star \star}$ & 337 & $652^{\star \star \star}$ & 344 \\
\hline Female: $31-50$ years $(n 812,30 \%)$ & 220 & 82 & $1506^{\star \star \star}$ & 814 & $1533^{\star \star \star}$ & 805 & $659^{\star * *}$ & 408 & $675^{\star \star \star}$ & 404 \\
\hline Male: $51-70$ years $(n 631,25 \%)$ & 287 & 113 & $1654^{\star \star \star}$ & 767 & $1702^{\star \star \star}$ & 762 & $702^{\star \star \star}$ & 372 & $727^{\star \star \star}$ & 370 \\
\hline Female: $51-70$ years $(n 1065,33 \%)$ & 238 & 86 & $1589^{\star \star \star}$ & 1014 & $1623^{\star \star \star}$ & 1002 & 299.5 & 329.4 & $688^{\star \star \star}$ & 506 \\
\hline Male: 71 years and over $(n 372,25 \%)$ & 268 & 98 & $1644^{\star \star \star}$ & 885 & $1703^{\star \star \star}$ & 886 & 291.5 & 308.7 & $704^{\star \star \star}$ & 432 \\
\hline Female: 71 years and over $(n 838,32 \%)$ & 214 & 85 & $1560^{\star \star \star}$ & 1042 & $1595^{\star \star \star}$ & 1035 & 286.3 & 351.5 & $685^{\star \star \star}$ & 517 \\
\hline Overall $(n 8390,26 \%)$ & 235 & 95 & $1344^{\star \star \star}$ & 824 & $1395^{\star \star \star}$ & 819 & 274.3 & 238.1 & $546^{\star \star \star}$ & 395 \\
\hline \multicolumn{11}{|l|}{ Non-supplement users ( $n$ 24 386) } \\
\hline $1-3$ years $(n 1514)$ & 136 & 58 & 269 & 111 & 307 & 127 & 78 & 42 & 100 & 53 \\
\hline $4-8$ years $(n 1992)$ & 173 & 58 & 387 & 102 & 451 & 114 & 126 & 37 & 160 & 48 \\
\hline Male: $9-13$ years $(n 1618)$ & 212 & 70 & 470 & 127 & 543 & 138 & 152 & 46 & 191 & 56 \\
\hline Female: $9-13$ years $(n 1555)$ & 176 & 59 & 396 & 111 & 461 & 122 & 129 & 35 & 164 & 40 \\
\hline Male: $14-18$ years ( $n$ 1995) & 252 & 98 & 565 & 180 & 653 & 205 & 186 & 59 & 231 & 75 \\
\hline Female: $14-18$ years ( $n$ 1916) & 193 & 77 & 426 & 146 & 487 & 171 & 138 & 51 & 171 & 67 \\
\hline Male: $19-30$ years $(n$ 1491) & 269 & 98 & 568 & 159 & 644 & 165 & 177 & 55 & 215 & 65 \\
\hline Female: $19-30$ years ( $n$ 1402) & 209 & 83 & 417 & 136 & 465 & 147 & 122 & 50 & 148 & 64 \\
\hline Male: $31-50$ years $(n$ 2055) & 262 & 88 & 527 & 163 & 594 & 180 & 155 & 63 & 189 & 76 \\
\hline Female: $31-50$ years $(n 1874)$ & 231 & 100 & 419 & 138 & 464 & 142 & 111 & 37 & 135 & 44 \\
\hline Male: $51-70$ years $(n$ 1919) & 253 & 101 & 445 & 139 & 498 & 151 & 114 & 46 & 140 & 58 \\
\hline Female: $51-70$ years ( $n$ 2135) & 215 & 77 & 378 & 103 & 418 & 116 & 94 & 26 & 116 & 40 \\
\hline Male: 71 years and over $(n 1148)$ & 208 & 79 & 371 & 116 & 418 & 131 & 97 & 42 & 122 & 53 \\
\hline Female: 71 years and over $(n$ 1772) & 185 & 68 & 317 & 97 & 352 & 107 & 78 & 37 & 97 & 46 \\
\hline Overall $(n 24386)$ & 228 & 95 & 442 & 168 & 503 & 182 & 112 & 55 & 600 & 399 \\
\hline
\end{tabular}

DFE, dietary folate equivalent.

${ }^{* * \star} P<0.001$ compared with non-supplement users.

‡ Overage is defined as the potential extra amount of folic acid added to a product during fortification by the food manufacturer to prevent decay/loss during shelf life/storage.

$\S$ Percentages in brackets reflect the proportion of supplement users in each age group. 
Table 2. Proportion of folic acid supplement users* from the Canadian Community Health Survey, cycle 2.2 (CCHS 2.2), and the Canadian Health Measures Survey (CHMS)

\begin{tabular}{|c|c|c|c|c|}
\hline \multirow[b]{2}{*}{ Life-stage groups } & \multicolumn{2}{|c|}{ CCHS $2.2(\%)$} & \multicolumn{2}{|c|}{ CHMS combined cycles (\%) } \\
\hline & Males ( $n$ 2612) & Females ( $n$ 3932) & Males $(n$ 1556) & Females ( $n$ 1978) \\
\hline $1-3$ years & \multicolumn{2}{|c|}{$28 \cdot 4$} & \multicolumn{2}{|c|}{ - } \\
\hline $4-8$ years & \multicolumn{2}{|c|}{38.4} & \multicolumn{2}{|c|}{28.5} \\
\hline $9-13$ years & $22 \cdot 2$ & 21.5 & $17 \cdot 5$ & $20 \cdot 1$ \\
\hline $14-18$ years & $12 \cdot 8$ & $15 \cdot 1$ & 11.5 & 14.5 \\
\hline $19-30$ years & $17 \cdot 4$ & $24 \cdot 4$ & $13 \cdot 1$ & $18 \cdot 6$ \\
\hline $31-50$ years & $20 \cdot 8$ & $30 \cdot 2$ & $18 \cdot 4$ & $27 \cdot 0$ \\
\hline $51-70$ years & $24 \cdot 7$ & 33.3 & $22 \cdot 8$ & 28.7 \\
\hline 71 years and over & 24.5 & $32 \cdot 1$ & $26 \cdot 0$ & $38 \cdot 1$ \\
\hline Overall & \multicolumn{2}{|c|}{$24 \cdot 7$} & \multicolumn{2}{|c|}{$22 \cdot 1$} \\
\hline
\end{tabular}

* Reported vitamin or supplements use in the $30 \mathrm{~d}$ before interview.
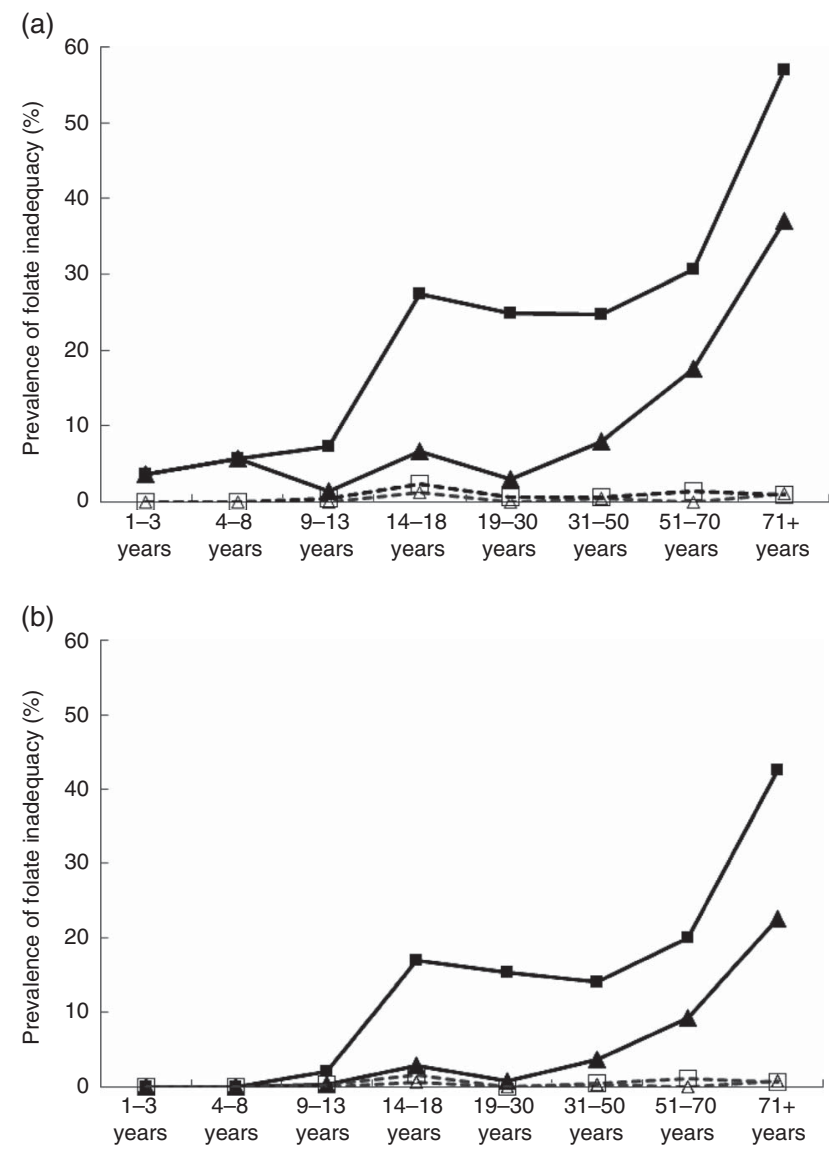

Fig. 1. Prevalence of folate inadequacy by dietary reference intakes life-stage group based on intake levels below the estimated average requirement (EAR) by sex and folic acid (FA) supplement use based on general fortification (a) and based on general fortification plus overage (b). Overage is defined as the potential extra amount of FA added to a product during fortification by the food manufacturer to prevent decay/loss during shelf life/storage. a: $\_$, Male non-supplement user

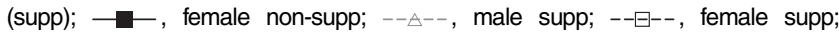
b: $\_$, male non-supp+overage; - - , female non-supp+overage; ----- , male supp+overage; -- $\square--$, female supp+overage. EAR values $(\mu \mathrm{g})$ : $1-3$ years (120), 4-8 years (160), 9-13 years (250), 14-18 years $(330), 19+$ years (320) (source: Institute of Medicine).

Among supplement users, the POFI was also very low $(<5 \%)$ for all adolescent and adult DRI life-stage groups, regardless of calculation method. Among non-supplement users, however, the POFI increased with age and was highest among older adults $>50$ years, with the highest proportion occurring in females $>70$ years. When POFI was estimated using only folate from unfortified food sources, inadequacy levels were very high, ranging from $38 \%$ to a staggering $94 \%$ among females aged 14-18 years (results not shown). When estimated with overage factors, POFI was low among supplement users $(<2 \%)$, but rose to $43 \%$ in older females who did not report supplement use. In addition, almost one-quarter (23\%) of older males aged 70 years and above had intakes below the EAR (Fig. 1(b)).

\section{Assessment of the prevalence of folate inadequacy: erythrocyte folate}

Results from the observed erythrocyte folate concentrations showed that folate deficiency among Canadians (erythrocytes folate $<305 \mathrm{nmol} / \mathrm{l})$ was virtually non-existent $(<1 \%)$, regardless of whether or not the conversion method was used. This level ( $305 \mathrm{nmol} / \mathrm{l}$ ) has been deemed by the IOM to denote a deficiency state, based on its association with megaloblastic anaemia ${ }^{(49,50)}$. Among WCBA, approximately 23\% showed erythrocyte folate concentrations below those deemed 'optimal' for maximal NTD risk reduction $(<906 \mathrm{nmol} / \mathrm{l})$.

\section{Assessment of the prevalence of folate overconsumption: dietary folate}

The UL for folate established by the IOM are based on the DFE intake of synthetic forms of folate (i.e. FA). Intake estimates to be compared against the UL do not include natural sources of folate, because high intakes from food have not been linked to adverse health effects ${ }^{(15)}$. Among non-supplement users, intake above the UL was virtually non-existent, even with overage additions taken into account (Figs. 2(a) and (b)). Among supplement users, however, the percentage with FA intakes above the UL was highest in respondents $>13$ years of age, regardless of sex, and the prevalence rate of folate above UL reached $18 \%$ among male supplement users aged 14-18 years when overage factors were considered.

\section{Assessment of the prevalence of folate over-concentration: erythrocyte folate}

High erythrocyte folate concentrations do exist among certain subgroups of the Canadian population (Fig. 3). Using the previously mentioned lower cut-off values determined by 

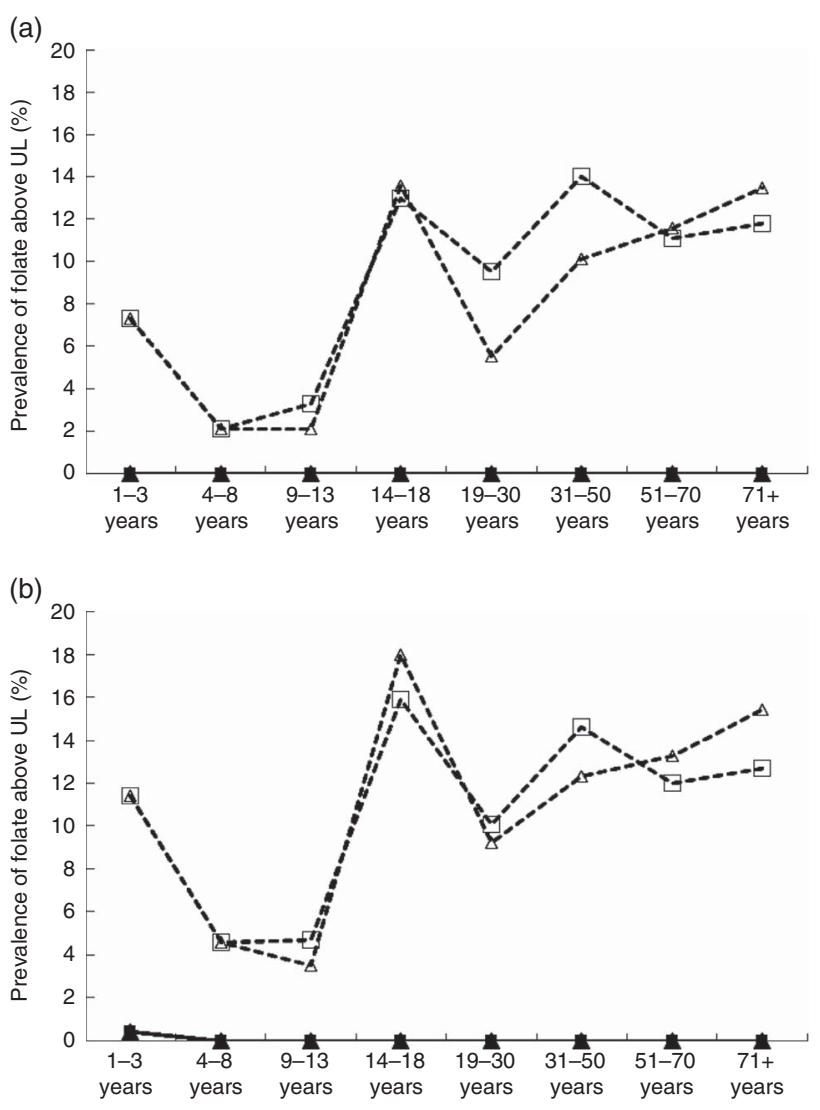

Fig. 2. Prevalence of folic acid overconsumption by dietary reference intakes life-stage group based on intake levels above the tolerable upper intake level (UL) by sex and folic acid (FA) supplement use based on general fortification (a) and based on general fortification plus overage (b). Overage is defined as the potential extra amount of FA added to a product during fortification by the food manufacturer to prevent decay/loss during shelf life/storage. a: $\_$, Male non-supplement user (supp); --A--, male supp; -- --- , female supp; $-\longrightarrow$, female non-supp; b: $\_-$, male non-supp + overage; --A--, male supp + overage; -- $\square--$, female supp +overage; $-\longrightarrow$, female non-supp +overage. UL values $(\mu \mathrm{g})$ : $1-3$ years (300), 4-8 years (400), 9-13 years (600), 14-18 years (800), 19+ years (1000) (source: Institute of Medicine).

Colapinto et al. ${ }^{(49)}$, in conjunction with their proposed conversion method, almost $30 \%$ of female supplement users and $20 \%$ of male supplement users had blood folate concentrations $>1450 \mathrm{nmol} / \mathrm{l}$ compared with 10 and $6 \%$ of female and male non-supplement users, respectively. Using the middle level cut-off, 12 and $7 \%$ of female and male supplement users had blood folate concentrations $>1800 \mathrm{nmol} / 1$, compared with 2.5 and $1 \%$ of non-supplement users of the corresponding sex. Using the highest cut-offs, $4 \%$ of supplement using Canadians had blood folate levels $>2150 \mathrm{nmol} / \mathrm{l}$, compared with $<1 \%$ of their non-supplement using counterparts (results not shown).

\section{Discussion}

\section{Folate inadequacy}

The results of this study demonstrate that mandatory folate fortification has led to significant improvements in the overall intake of folate in the Canadian population, as the overall POFI (dietary and clinical) was found to be low. However,

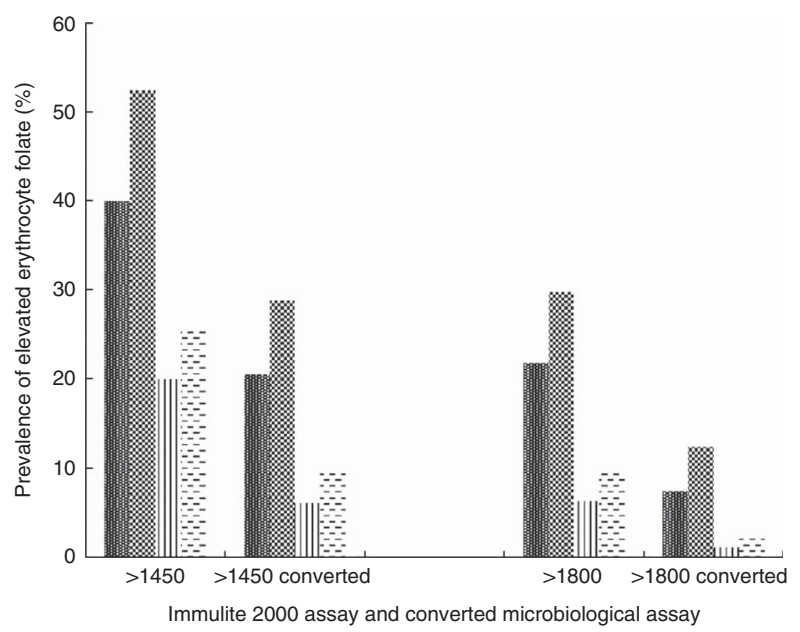

Fig. 3. Prevalence of elevated erythrocyte folate concentrations by sex and supplement use status based on proposed cut-offs, as well as by conversion factor. Converted microbiological assay concentration $=-22.95 \times(0.81) \times$ Immulite 2000 assay concentrations ${ }^{(49)}$. 晅, Male supplement user (supp); 叉, female supp; 血, male non-supp; $\mathrm{\theta}^{-}$, non-female supp.

non-supplement users, particularly adults $>70$ years, may still be at risk for folate deficiency. Rates of POFI were comparable with previous studies by Bailey et $a l .{ }^{(51)}$ and Shakur et $a l^{(34)}$, with the highest POFI occurring among females $>70$ years, and may reflect the fact that older adults tend to consume less food overall. These results are similar to recent findings that showed up to one-third of elderly Irish men and women ( $>64$ years) did not meet the requirements for optimal intake ${ }^{(52)}$. Overall, estimates of total DFE intake were comparable with those previously published by Shakur et al. using results from the CCHS 2.2(34). Any small differences may be attributed to disparities in inclusion criteria as well as the fact that their study used the CCHS 2.2 Master File ( $n$ 35107), whereas this study utilised the Share File ( $n$ 33469), which is a slightly smaller subset of the Master file containing data on respondents willing to share responses with the survey Share partners ${ }^{(53)}$. Similarly, overall prevalence of erythrocyte folate concentrations $<305 \mathrm{nmol} / 1$ (used to estimate deficiency) as well as that of WCBA were similar to the results by Colapinto et al. ${ }^{(47)}$ using cycle 1 of the CHMS. The unique contribution of this study is that it utilised both the 24-h dietary recall data and the blood sample estimates to assess folate inadequacy. POFI based on 24-h dietary recall data were shown to be comparable with the estimates based on erythrocyte folate data. Results from both surveys showed 24.5 and $23 \%$ of WCBA below the optimal level of folate for maximal NTD risk reduction, indirectly validating the 24 -h recall method on estimating nutrient intake and status.

\section{Supplement use and folate intake}

Results from both the CCHS and the CHMS showed similar proportions of FA supplement users. Although the highest user rate appeared among respondents aged 4-8 years in the CCHS, the CHMS results showed that females $>70$ years had the highest reported rates of FA supplement use, followed by females aged 51-70 years and children under 8 years of age. 
In our study, the prevalence of supplement use among children aged 4-8 years (38\%) was comparable with the $36 \%$ supplement use prevalence observed in US children $\leq 13$ years of age based on NHANES data ${ }^{(51)}$, although slightly lower rates (29\%) were observed among children in the CHMS. This difference could be due to the smaller sample size of children in the CHMS. Our FA supplement use percentages also corresponded with previous CCHS 2.2 analyses ${ }^{(34)}$.

CCHS supplement users were unlikely to be folate deficient. However, as demonstrated by our analysis, it is critical that supplement users should be observed separately from non-supplement users, as this particular group is at an increased risk for possible overconsumption ${ }^{(54)}$. Intakes of folate in nonsupplement using adults in this study were higher than those generated from the NHANES 1999-2000 data ( $\geq 20$ years, $n$ 2121), perhaps due to demographic differences (such as including a younger age range of 2-18 years) or variations in food sources consumed by both populations ${ }^{(55)}$. Conversely, mean folate intakes from this study were somewhat lower than those previously reported in a study that used data from NHANES 2003-2006 to examine folate intake in Americans $\leq 13$ years of age $^{(7,51)}$. Paediatric intakes from this study are similar to intakes in Ontario pre-school children aged $3-5$ years ( $n$ 254), whose average intake of DFE was calculated to be $336 \mu \mathrm{g}^{(56)}$. Results from both surveys showed that $<25 \%$ of WCBA consumed FA-containing supplements, similar to the findings of Shuaibi et $a l^{(14)}$ who observed that only $26 \%$ of females aged 18-25 years used supplements. However, although supplement use was found to ameliorate folate inadequacy, it should be noted that CCHS 2.2 data also provide evidence that certain subgroups are consuming levels of FA above the UL.

\section{Folate consumption above the tolerable upper limit and elevated erythrocyte folate concentration}

The pooled CHMS data show that certain Canadians have elevated erythrocyte folate concentrations. Rates of intake above the UL for FA reached $18 \%$ in male supplement users aged 14-18 years (when potential overages were accounted for), and up to $25 \%$ of supplement users have elevated blood folate levels. In light of a recent speculation that high levels of FA may be potentially linked to increased cancer risk and cognitive decline, this may be cause for concern. Although there are little data on the implications of FA toxicity among growing children and adolescents, some researchers have suggested consideration be given to removing FA from supplements designed for children and men ${ }^{(36)}$.

Previous studies have suggested that FA intakes greater than the UL are considered safe and that care must be taken when interpreting risks of intakes above the $\mathrm{UL}^{(54)}$, because the body of literature used to derive UL for most nutrients is limited ${ }^{54,57)}$. However, recent observations from the Framingham study demonstrate that regular users of vitamin supplements have a mean concentration of unmetabolised FA in fasting plasma that is approximately $40 \%$ higher than non-users ${ }^{(17)}$. Moreover, $>80 \%$ of regular vitamin users have detectable levels of unmetabolised FA in their plasma ${ }^{(17,32)}$, which may decrease the cytotoxicity of lymphocytes thought to play a role in the destruction of neoplastic cells in older women ${ }^{(32)}$ - a group that this study shows is consuming FA above the UL. This same population is also more susceptible to vitamin $\mathrm{B}_{12}$ deficiency ${ }^{(9)}$ and potentially masking this problem with adequate FA intake $e^{(9,33,58)}$. A unique contribution of this study is the estimation and comparison of the prevalence of folate overconsumption and elevated folate status. The proportion of folate consumption above the UL based on dietary intake data was higher than the estimates based on elevated erythrocyte folate concentrations using the middle or higher cut-offs, but lower than the estimates using the lower cut-off. Nevertheless, both estimates (24-h dietary recall and erythrocyte folate concentration) revealed that higher-than-optimal folate intakes and elevated blood concentrations exist in the Canadian population.

The data presented in this report are subject to a few limitations. In the CCHS 2.2, only a subset of respondents provided second-day dietary intake data on which usual intake estimates were based, although the use of SIDE software aids in remedying this by using the subset of 2 nd-d recalls to adjust variance estimates and generate stable usual intake estimates for groups. Although the use of overage factors have been based on a rather limited amount of fortified foods ( $n$ 92), the resulting overage percentages reported are comparable with those determined previously by other authors ${ }^{(34-36)}$. In addition, the CCHS 2.2 is a self-reported survey, and non-sampling errors such as non-response, recall bias and social desirability may affect the validity of results. Although the five-step multiple-pass method utilised during the 24-h recall has been shown to enhance accuracy and assist the respondent in remembering what and how much food they consumed ${ }^{(59)}$, it has been reported that the average under-reporting of energy intake in the CCHS 2.2 is estimated at $10 \%$, with a greater underreporting rate among respondents who were overweight or obese, adults compared with teenagers and women compared with men ${ }^{(60)}$. Finally, a limitation of this study is that there does not exist a universal cut-off for elevated erythrocyte folate, and it must be noted that using higher cut-offs may discount individuals with potentially high folate statuses. Consequently, using slightly higher cut-offs will also provide information on those subgroups in which high folate status is most prevalent.

The reference range of erythrocyte folate varies by age, but generally falls between 317 and $1422 \mathrm{nmol} / \mathrm{l}^{(61)}$. Researchers have used quantiles of study populations to postulate cut-offs for erythrocyte folate ${ }^{(47,62,63)}$. MacFarlane et al. ${ }^{(62)}$ proposed an erythrocyte folate cut-off of $1090 \mathrm{nmol} / 1$ based on data from Quinlivan \& Gregory ${ }^{(63)}$, who determined the relationship between dietary folate intake and erythrocyte folate to be $2.1 \mathrm{mg}$ $\mathrm{DFE} / 1090 \mathrm{nmol} / \mathrm{l}$. This reflects a combined intake of $0.4 \mathrm{mg} \mathrm{DFE}$ (based on RDA) and a $1 \mathrm{mg}$ FA supplement (bioavailability $1.7 \mathrm{mg}$ ). Using results from the CHMS 2007-2009, they observed that $63.5 \%$ of Canadians had erythrocyte folate levels above the $1090 \mathrm{nmol} / 1$ cut-off $^{(62)}$. However, a more stringent cut-off of $1360 \mathrm{nmol} / \mathrm{l}$, which was based on the concentration at the 97th percentile of the NHANES 1988-2010 suggested by Colapinto, reduced this proportion to $40 \%{ }^{(47)}$. Pfeiffer et al. ${ }^{(64)}$ suggested three cut-offs using NHANES data (erythrocyte folate 
concentrations obtained via a microbiological assay) using upper erythrocyte folate concentrations at the 90th, 95th and 97.5th percentiles of 1820, 2140 and $2490 \mathrm{nmol} / \mathrm{l}$, respectively. Colapinto mimicked these percentiles using converted CHMS erythrocyte folate data, obtaining slightly lower cut-offs than Pfeiffer. For the purpose of practicality, this study uses the cut-offs established by Colapinto et $a l^{(49)}$, which were based on post-fortification NHANES data of 1450, 1800 and $2150 \mathrm{nmol} / 1$ that occurred at regular intervals $(350 \mathrm{nmol} / 1$ apart $)$ within this range of postulated cut-offs. In comparison with MacFarlane's results, using the conversion factor and more stringent high erythrocyte folate cutoffs decreased the proportion of Canadians with high erythrocyte folate status to $16 \cdot 4 \%{ }^{(49)}$. It is evident that both fortified foods and FA supplements are increasing the erythrocyte folate status of Canadians. With this in mind, it is possible that a future erythrocyte folate cut-off based on these higher population intakes may be established. However, any cut-offs established in the future must be treated carefully and be paired with extensive research and long-term studies, particularly in light of recent data showing cognitive impairment associated with high plasma folate ${ }^{(18)}$. It will be necessary to strike a balance between establishing a cut-off that reflects the dietary changes of the population at hand, but also protects this same population from adverse health effects.

A strength of this project is that the POFI as well as toxicity was based on a combination of dietary, supplemental and clinical measures of status. The results of both survey data showed comparable results on folate intakes below EAR and above UL as well as those above the recommended cut-offs. Therefore, the results of this study are generalisable to other similar populations with a similar food supply environment.

\section{Conclusions}

Results of several studies leave little doubt that mandated folate fortification has led to significant improvements in the overall intake of folate in North America and to impressive decreases in the incidence of NTD. Ideally, one's diet should provide sufficient intake of folate, and data provide evidence that, although folate intake of Canadians has increased after fortification, consumption of folate from naturally occurring and fortified food sources may not be enough to achieve the desired levels of folate as recommended by the IOM, particularly for WCBA. In light of our results that $23 \%$ of WCBA had erythrocyte folate concentrations below those deemed 'optimal' for maximal NTD risk reduction, it should be suggested that females aged 15-45 years who do not consume a FA-containing supplement on a daily basis should start to do so if a pregnancy is expected or planned.

Although previous studies have suggested that FA intakes above the UL are considered safe, a careful evaluation of the risks and benefits of food fortification, particularly above mandated levels, is necessary. Recent research shows cognitive impairment to be associated with high erythrocyte folate, and emerging animal studies highlight other adverse outcomes linked with excessive FA. In light of increasing evidence suggesting that certain subgroups of the population may be at risk by being subjected to high levels of FA, it seems prudent that persons of all ages who may be considering a FA-containing supplement should be cautioned about the potential risk involved with FA consumption above the UL. In particular, it may be suggested that vitamins targeted towards children, men, women past childbearing age and older adults not include FA. Future research and studies monitoring excessive folate intake are imperative in order to find a healthy balance to achieve optimal intake, and well-designed longitudinal studies are needed to draw definitive conclusions regarding the adverse effects of consuming excess FA. Consequently, it is important to universally define high blood folate status in order to better inform health professionals as well as the public about the benefits and risks of increased FA intake and elevated status.

\section{Acknowledgements}

The authors thank Dr Ian Clara and Kelly Cranswick at the Manitoba Research Data Centre for their statistical help and support for this project. The authors thank Dr Amanda MacFarlane at Nutrition Research Division, Health Canada, for her thorough review and constructive suggestions for this paper and Linda Greene-Finestone from the Public Health Agency of Canada for her assistance and suggestions.

Funding for this study was provided by the Public Health Agency of Canada.

All the authors were responsible for the study design and assisted in manuscript revision. M. d. G. and N. Y. were responsible for project conception. A. N. M. and N. Y. were responsible for data analyses and interpretation of the results. A. N. M., N. Y., H. M. A. and M. d. G. were responsible for drafting the manuscript and critical revision. The interpretation of results or points of view expressed in this paper are those of the authors and do not necessarily represent the opinions or position of the Public Health Agency of Canada.

The authors declare that there are no conflicts of interest.

\section{References}

1. National Institutes of Health. Office of Dietary Supplements (2012) Folate: dietary supplement fact sheet. http://ods. od.nih.gov/factsheets/Folate-HealthProfessional (accessed January 2016).

2. Murphy M, Whiteman D, Stone D, et al. (2000) Dietary folate and the prevalence of neural tube defects in the British Isles: the past two decades. BJOG 107, 885-889.

3. Laurence KM, James N, Miller MH, et al. (1981) Double-blind randomised controlled trial of folate treatment before conception to prevent recurrence of neural-tube defects. Br Med J (Clin Res Ed) 282, 1509-1511.

4. Wilson RD, Johnson J, Wyatt P, et al. (2007) Pre-conceptional vitamin/folic acid supplementation 2007: the use of folic acid in combination with a multivitamin supplement for the prevention of neural tube defects and other congenital anomalies. J Obstet Gynaecol Can 29, 1003-1026.

5. Berry RJ, Li Z, Erickson JD, et al. (1999) Prevention of neural-tube defects with folic acid in China. N EnglJ Med 341, $1485-1490$

6. Milunsky A, Jick H, Jick SS, et al. (1989) Multivitamin/ folic acid supplementation in early pregnancy reduces the prevalence of neural tube defects. JAMA $\mathbf{2 6 2}$, $2847-2852$ 
7. Bailey LB \& Berry RJ (2005) Folic acid supplementation and the occurrence of congenital heart defects, orofacial clefts, multiple births, and miscarriage. Am J Clin Nutr 81, 1213S-1217S.

8. Centres for Disease Control and Prevention (2012) Folic acid recommendations. http://www.cdc.gov/ncbddd/folicacid/ recommendations.html (accessed January 2016).

9. Liu S, West R, Randell E, et al. (2004) A comprehensive evaluation of food fortification with folic acid for the primary prevention of neural tube defects. BMC Pregnancy Childbirth 4, 20.

10. Choumenkovitch SF, Selhub J, Wilson PW, et al. (2002) Folic acid intake from fortification in United States exceeds predictions. J Nutr 132, 2792-2798.

11. House JD, March SB, Ratnam S, et al. (2006) Improvements in the status of folate and cobalamin in pregnant Newfoundland women are consistent with observed reductions in the incidence of neural tube defects. Can J Public Health 97, 132-135.

12. De Wals P, Tairou F, Van Allen MI, et al. (2007) Reduction in neural-tube defects after folic acid fortification in Canada. $N$ Engl J Med 357, 135-142.

13. Ray JG, Vermeulen MJ, Boss SC, et al. (2002) Increased red cell folate concentrations in women of reproductive age after Canadian folic acid food fortification. Epidemiology 13, 238-240.

14. Shuaibi AM, House JD \& Sevenhuysen GP (2008) Folate status of young Canadian women after folic acid fortification of grain products. J Am Diet Assoc 108, 2090-2094.

15. Institute of Medicine (2000) Dietary reference intakes for thiamin, riboflavin, niacin, vitamin $\mathrm{B}_{6}$, folate, vitamin $\mathrm{B}_{12}$, pantothenic acid, biotin, and choline. http://www.iom.edu/Reports/2000/DietaryReference-Intakes-for-Thiamin-Riboflavin-Niacin-Vitamin-B6Folate-Vitamin-B12-Pantothenic-Acid-Biotin-and-Choline.aspx (accessed February 2016).

16. University of Maryland Medical Centre (2013) Vitamins. http:// www.umm.edu/patiented/articles/what_vitamins_000039_1. htm (accessed February 2016).

17. Kalmbach RD, Choumenkovitch SF, Troen AM, et al. (2008) Circulating folic acid in plasma: relation to folic acid fortification. Am J Clin Nutr 88, 763-768.

18. Selhub J \& Rosenberg IH (2016) Excessive folic acid intake and relation to adverse health outcomes. Biochimie 126, 71-78.

19. Smith AD, Refsum H, Selhub J, et al. (2016) Decision on folic acid fortification in Europe must consider both risks and benefits. BMJ 352, i734.

20. Baik H \& Russell R (1999) Vitamin $B_{12}$ deficiency in the elderly. Annu Rev Nutr 19, 357-377.

21. Yajnik C, Deshpande S, Jackson A, et al. (2008) Vitamin $B_{12}$ and folate concentrations during pregnancy and insulin resistance in the offspring: the Pune Maternal Nutrition Study. Diabetologia 51, 29-38.

22. Sawaengsri H, Wang J, Reginaldo C, et al. (2016) High folic acid intake reduces natural killer cell cytotoxicity in aged mice. J Nutr Biochem 30, 102-107.

23. Meadows DN, Bahous RH, Best AF, et al. (2015) High dietary folate in mice alters immune response and reduces survival after malarial infection. PLOS ONE 10, e0143738.

24. Mason JB, Dickstein A, Jacques PF, et al. (2007) A temporal association between folic acid fortification and an increase in colorectal cancer rates may be illuminating important biological principles: a hypothesis. Cancer Epidemiol Biomarkers Prev 16, 1325-1329.

25. Cole BF, Baron JA, Sandler RS, et al. (2007) Folic acid for the prevention of colorectal adenomas: a randomized clinical trial. JAMA 297, 2351-2359.

26. Wu K, Platz EA, Willett WC, et al. (2009) A randomized trial on folic acid supplementation and risk of recurrent colorectal adenoma. Am J Clin Nutr 90, 1623-1631.
27. Vollset SE, Clarke R, Lewington S, et al. (2013) Effects of folic acid supplementation on overall and site-specific cancer incidence during the randomised trials: meta-analyses of data on 50,000 individuals. Lancet 381, 1029-1036.

28. Kim Y (2006) Does a high folate intake increase the risk of breast cancer? Nutr Rev 64, 468-475.

29. Siu MK, Kong DS, Chan HY, et al. (2012) Paradoxical impact of two folate receptors, FR $\alpha$ and RFC, in ovarian cancer: effect on cell proliferation, invasion and clinical outcome. PLOS ONE 7, e47201.

30. Morris MS, Jacques PF, Rosenberg IH, et al. (2010) Circulating unmetabolized folic acid and 5-methyltetrahydrofolate in relation to anemia, macrocytosis, and cognitive test performance in American seniors. Am J Clin Nutr 91, 1733-1744.

31. Mason JB (2009) Folate, cancer risk, and the Greek god, Proteus: a tale of two chameleons. Nutr Rev 67, 206-212.

32. Troen AM, Mitchell B, Sorensen B, et al. (2006) Unmetabolized folic acid in plasma is associated with reduced natural killer cell cytotoxicity among postmenopausal women. J Nutr 136, 189-194.

33. Selhub J, Morris MS, Jacques PF, et al. (2009) Folate-vitamin B-12 interaction in relation to cognitive impairment, anemia, and biochemical indicators of vitamin B-12 deficiency. Am J Clin Nutr 89, 702S-706S.

34. Shakur YA, Garriguet D, Corey P, et al. (2010) Folic acid fortification above mandated levels results in a low prevalence of folate inadequacy among Canadians. Am J Clin Nutr $\mathbf{9 2}$, 818-825.

35. Quinlivan EP \& Gregory IJ (2003) Impact of food fortification on folic acid intake in Canada (Letter to editor). Can J Public Health 94, 154.

36. Shakur YA, Rogenstein C, Hartman-Craven B, et al. (2009) How much folate is in Canadian fortified products 10 years after mandated fortification? Can J Public Health 100, 281-284.

37. Lowe H, Cusano NE, Binkley N, et al. (2010) Vitamin D toxicity due to a commonly available 'over the counter' remedy from the Dominican Republic. J Clin Endocrinol Metabol 96, 291-295.

38. Mayo-Wilson E, Imdad A, Herzer K, et al. (2011) Vitamin A supplements for preventing mortality, illness, and blindness in children aged under 5: systematic review and meta-analysis. BMJ 343, d5094.

39. Health Canada (2008) Canadian Community Health Survey, Cycle 2.2, Nutrition (2004): income related household food security in Canada. http://www.hc-sc.gc.ca/fn-an/surveill/ nutrition/commun/income_food_sec-sec_alim-eng.php (accessed February 2016).

40. Health Canada (2012) Canadian Community Health Survey 2.2, Nutrition focus. http://www.hc-sc.gc.ca/fn-an/surveill/ nutrition/commun/cchs_focus-volet_escc-eng.php (accessed February 2016).

41. Garriguet D (2016) Canadian Community Health Survey, Cycle 2.2. http://www.utoronto.ca/rdc/files/CCHS_2.2_ Presentation.pdf (accessed February 2016).

42. Bolger C (2008) Canadian Health Measures Survey. Health reports, Statistics Canada. http://ir.lib.uwo.ca/rdchealthcon ference/preconference/program/2/ (accessed February 2016).

43. Tremblay M, Wolfson M \& Connor Gorber S (2007) Canadian Health Measures Survey: rationale, background and overview. Health Rep 18, Suppl., 7-20.

44. Suitor CW \& Bailey LB (2000) Dietary folate equivalents: interpretation and application. J Am Diet Assoc 100, 88-94.

45. Dodd KW, Guenther PM, Freedman LS, et al. (2006) Statistical methods for estimating usual intake of nutrients and foods: a review of the theory. J Am Diet Assoc 106, 1640-1650. 
46. Statistics Canada (2007) Canadian Community Health Survey (CCHS) Cycle 2.2 - Nutrition; Software for Intake Distribution Estimation (SIDE) Documentation, Statistics Canada. http:// www23.statcan.gc.ca/imdb-bmdi/document/5049_D22_T9_ V1-eng.pdf (accessed February 2016).

47. Colapinto CK, O'Connor DL \& Tremblay MS (2011) Folate status of the population in the Canadian Health Measures Survey. CMAJ 183, E100-E106.

48. Colapinto CK, Tremblay MS, Aufreiter S, et al. (2014) The direction of the difference between Canadian and American erythrocyte folate concentrations is dependent on the assay method employed: a comparison of the Canadian Health Measures Survey and National Health and Nutrition Examination Survey. Br J Nutr 112, 1873-1881.

49. Colapinto CK, O'Connor DL, Dubois L, et al. (2015) Prevalence and correlates of high red blood cell folate concentrations in the Canadian population using 3 proposed cut-offs. Appl Physiol Nutr Metab 40, 1025-1030.

50. Crider KS, Devine O, Hao L, et al. (2014) Population red blood cell folate concentrations for prevention of neural tube defects: Bayesian model. BMJ 349, g4554.

51. Bailey RL, Dodd KW, Gahche JJ, et al. (2010) Total folate and folic acid intake from foods and dietary supplements in the United States: 2003-2006. Am J Clin Nutr 91 231-237.

52. Power S, Jeffery I, Ross R, et al. (2014) Food and nutrient intake of Irish community-dwelling elderly subjects: who is at nutritional risk? J Nutr Health Aging 18, 561-572.

53. Health Canada (2010) Canadian Community Health Survey: Cycle 2.2: A guide to accessing and interpreting the data. http:// www.hc-sc.gc.ca/fn-an/surveill/nutrition/commun/cchs_guide_ escc-eng.php (accessed February 2016).

54. Shakur YA, Tarasuk V, Corey P, et al. (2012) A comparison of micronutrient inadequacy and risk of high micronutrient intakes among vitamin and mineral supplement users and nonusers in Canada. J Nutr 142, 534-540.
55. Dietrich M, Brown CJ \& Block G (2005) The effect of folate fortification of cereal-grain products on blood folate status, dietary folate intake, and dietary folate sources among adult non-supplement users in the United States. J Am Coll Nutr 24, 266-274.

56. Hennessy-Priest K, Mustard J, Keller H, et al. (2009) Folic acid food fortification prevents inadequate folate intake among preschoolers from Ontario. Public Health Nutr 12, 1548-1555.

57. Carriquiry AL \& Camano-Garcia G (2006) Evaluation of dietary intake data using the tolerable upper intake levels. J Nutr 136, 507S-513S.

58. Morris MS, Jacques PF, Rosenberg IH, et al. (2007) Folate and vitamin B-12 status in relation to anemia, macrocytosis, and cognitive impairment in older Americans in the age of folic acid fortification. Am J Clin Nutr 85, 193-200.

59. Moshfegh AJ, Rhodes DG, Baer DJ, et al. (2008) The US Department of Agriculture automated multiple-pass method reduces bias in the collection of energy intakes. Am J Clin Nutr 88, 324-332.

60. Garriguet D (2008) Under-reporting of energy intake in the Canadian Community Health Survey. Health Rep 19, 37-45.

61. Fischbach FT \& Dunning MB (2009) A manual of laboratory and diagnostic tests. Philadelphia, PA: Lippincott Williams \& Wilkins.

62. MacFarlane AJ, Greene-Finestone LS \& Shi Y (2011) Vitamin B-12 and homocysteine status in a folate-replete population: results from the Canadian Health Measures Survey. Am J Clin Nutr 94, 1079-1087.

63. Quinlivan EP \& Gregory JF 3rd (2007) Reassessing folic acid consumption patterns in the United States (1999-2004): potential effect on neural tube defects and overexposure to folate. Am J Clin Nutr 86, 1773-1779.

64. Pfeiffer CM, Hughes JP, Lacher DA, et al. (2012) Estimation of trends in serum and RBC folate in the U.S. population from pre- to postfortification using assay-adjusted data from the NHANES 1988-2010. J Nutr 142, 886-893. 